\title{
The Dickeya dadantii biofilm matrix consists of cellulose nanofibres, and is an emergent property dependent upon the type III secretion system and the cellulose synthesis operon
}

Correspondence

Amy O. Charkowski

acharkowski@wisc.edu

Received 6 May 2011

Revised 22 June 2011

Accepted 24 June 2011

\author{
Courtney E. Jahn,† Dija A. Selimi,ł Jeri D. Barak and Amy O. Charkowski
}

Department of Plant Pathology, University of Wisconsin-Madison, Madison, WI 53706, USA

\section{INTRODUCTION}

Bacterial cellulose has been successfully used in a wide range of applications, including wound dressings, scaffolds for tissue growth, food additives, and flexible screen displays (Tabuchi, 2007). It is biocompatible and biodegradable, and can be modified by altering bacterial growth conditions or by adding compounds or polymers after it has been synthesized (Brown \& Laborie, 2007; Svensson et al., 2005; Wiegand et al., 2006). To this end, there is progress toward incorporating particles, enzymes, growth factors and antibiotics into bacterial cellulose scaffolds, which will improve their utility in tissue regeneration, biosensors and wound dressings (Phelps et al., 1994; Sharp et al., 2008; Tsioptsias \& Panayiotou, 2008). Although many bacterial species produce cellulose,

tPresent address: Department of Bioagricultural Sciences and Pest Management, Colorado State University, Fort Collins, CO, USA.

¥Present address: Center for Pre-Health Advising, University of Wisconsin-Madison, Madison, WI, USA.

Abbreviations: c-di-GMP, 3'-5'-cyclic diguanylic acid; SAL biofilm, surface-air-liquid interface biofilm; SEM, scanning electron microscopy; T3SS, type III secretion system. the soil bacterium Gluconacetobacter xylinum is the species most commonly used for industrial cellulose production.

Synthesis of bacterial cellulose, which has mainly been examined in G. xylinus, remains poorly understood. This bacterium produces cellulose fibres $3-8 \mathrm{~nm}$ thick from a linear array of pores along the sides of the bacterial cells (Bielecki et al., 2002). The array of pores secretes strands of cellulose that are crystallized into nanofibres upon secretion from the cell. G. xylinus cellulose production requires two polycistronic operons. One operon encodes a cellulose synthase subunit with a $3^{\prime}-5^{\prime}$-cyclic diguanylic acid (c-diGMP) binding domain $(b c s A)$, the beta subunit of the synthase $(b c s B)$, and two genes $(b c s C$ and $b c s D)$ implicated in the assembly of the glucan chains (Saxena et al., 1990, 1991, 1994). A second operon encodes a cellulase and a gene of unknown function (Standal et al., 1994). Homologous genes are found in some enterobacteria, but the operon has greatly diverged from that of G. xylinus, and an additional operon, $b c s E F G$, is required for cellulose synthesis in species with the second type of cellulose synthesis gene cluster (Solano et al., 2002). Recently, bcsQ (yhjQ), which is encoded upstream of $b c s A$, has been shown to be required for cellulose synthesis, and BscQ is located at the cell pole in Escherichia coli (Le Quéré \& Ghigo, 2009). 
The signal molecule c-di-GMP plays a role in cellulose synthesis. In enterobacteria, AdrA and/or YedQ, which are both predicted to be diguanylate cyclases, signal the cellulose synthesis machinery through an as-yet-undescribed mechanism (Da Re \& Ghigo, 2006; García et al., 2004; Kader et al., 2006). In some enterobacteria, such as Salmonella enterica, the curli regulator AgfD also regulates cellulose synthesis (Römling et al., 2000). However, this regulator is not widespread within the enterobacteria, and AgfD does not regulate cellulose in some enterobacterial strains that encode this gene (Da Re \& Ghigo, 2006).

The enterobacterial plant pathogen Dickeya dadantii forms two types of biofilm in culture: a surface-air-liquid interface biofilm (SAL biofilm) and a biofilm at the airliquid interface (pellicle) (Yap et al., 2005). The SAL biofilm and the pellicle are genetically and chemically distinct. Only the pellicle contains cellulose, and the type III secretion system (T3SS) and flagella are required only for the formation of a pellicle, and not a SAL biofilm (Jahn et al., 2008; Yap et al., 2005). D. dadantii is a broad-hostrange pathogen that causes wilt and rot diseases on many vegetable and ornamental crops. There are multiple stages in the $D$. dadantii life cycle in which biofilms could play a role, including pathogen survival in waterways and on plant debris, and during colonization of plant xylem vessels. $D$. dadantii is also an insect pathogen, and it is feasible that biofilms could play a role in its interactions with insects (Grenier et al., 2006).

We used microscopy and mutagenesis to examine $D$. dadantii cellulose synthesis, and found that $D$. dadantii produces a cellulose nanofibre decorated with regularly spaced beads, unlike any previously described biofilm matrix. To further explore how genes known to contribute to $D$. dadantii pellicle formation affected the structure of the cellulose nanofibres, we used microscopy and immunoassays to examine the pellicle matrix and composition in wild-type cells and in strains carrying mutations in gene clusters known to affect $D$. dadantii pellicle formation, including cellulose biosynthesis, the type III secretion system (T3SS), and flagella genes, as well as an orthologue of a gene known to regulate cellulose biosynthesis in S. enterica, adrA. Through these experiments, we found that mutation of the cellulose synthase $b c s A$ and of $b c s C$ reduces accumulation of the T3SS-secretion protein HrpN. In turn, mutation of HrpL, the sigma factor that controls the T3SS, abolishes synthesis of decorated cellulose nanofibres. Mutation of $a d r A$ did not eliminate cellulose synthesis, but did alter pellicle properties. We also found that beads of the reactive oxygen-scavenging secondary metabolite indigoidine produced by $D$. dadantii become embedded in the cellulose matrix.

\section{METHODS}

Bacterial strains and growth conditions. Wild-type $D$. dadantii and derivatives and $S$. enterica were grown in Luria-Bertani (LB) medium at 30 or $37^{\circ} \mathrm{C}$ for DNA isolation, for mutagenesis, and for starter cultures for biofilm experiments. SOBG was used to induce biofilm formation in D. dadantii (Yap et al., 2005). Bacterial growth in SOBG was measured both by monitoring the optical density of the cultures and by dilution plating from SOBG cultures. G. xylinus was grown in buffered S\&H medium (per litre: $5 \mathrm{~g}$ peptone, $5 \mathrm{~g}$ yeast extract, $2.7 \mathrm{~g} \mathrm{Na}_{2} \mathrm{HPO}_{4}, 1.15 \mathrm{~g}$ citric acid and $40 \mathrm{ml} 50 \%$ filtersterilized glucose, which was added after autoclaving the other medium components). S. enterica biofilm cultures were grown in LBNS (LB no salt) medium (per litre: $10 \mathrm{~g}$ tryptone, $5 \mathrm{~g}$ yeast extract). For pellicle assays, bacterial cultures were suspended in SOBG (D. dadantii), buffered S\&H medium or SOBG (G. xylinus), or LBNS (S. enterica) to approximately $10^{6}$ c.f.u. $\mathrm{ml}^{-1}$, and the cultures were incubated at $28{ }^{\circ} \mathrm{C}$ in stationary 13 or $20 \mathrm{~mm}$ glass tubes for up to 3 days. When required, antibiotics were used at the following concentrations (in $\mathrm{mg} \mathrm{ml}^{-1}$ ): nalidixic acid (50), ampicillin (100), chloramphenicol (50) and kanamycin (50). Strains used in this study are described in Table 1.

Mutant and plasmid construction. Primers were obtained from Integrated DNA Technologies (Table 2). To disrupt $a d r A$, a chloramphenicol-resistance cassette was ligated into the unique restriction site BglII in pGEM-T Easy:: adrA. Similarly, to disrupt $c e l Y$, a kanamycin-resistance cassette was ligated into the unique restriction site $X c m I$ of pGEM-T Easy::celY. The complete $b c s C$ coding region was removed with crossover PCR, and a fragment containing only the $b c s C$ flanking regions was inserted into pGEM-T Easy. Primers P0808 and P0809 amplify the region $1.5 \mathrm{~kb}$ upstream of $b c s C$, while P0810 and P0811 amplify the region $1.5 \mathrm{~kb}$ downstream of $b c s C$. Primers P0809 and P0810 have $19 \mathrm{bp}$ of overlapping nucleotide sequence; thus, crossover PCR with primers P0808 and P0811, with the flanking regions amplified by P0808/P0809 and P0810/P0811 as template, amplified a fragment with $b c s B$ adjacent to $b c s D$. This $3.0 \mathrm{~kb}$ crossover product was ligated into pGEM-T Easy. A chloramphenicol-resistance gene cassette was then ligated into a HindIII site engineered between $b c s B$ and $b c s D$ to construct pT $\Delta$ bcsC::Cm. These plasmids were electrotransformed into $D$. dadantii strains for allelic exchange following the methods described by Ried \& Collmer (1987). All mutations were confirmed by PCR and Southern blot analysis. Transformation, restriction endonuclease digestion and other DNA manipulations were performed essentially as described in Sambrook \& Russell (2001).

Cellulase assays. Cellulase assays were performed essentially as described in Yap et al. (2005), except that the incubation time was increased from $40 \mathrm{~min}$ to overnight. Pellicles were removed from 3 dayold biofilm-inducing cultures by pouring the culture into a Petri plate. The pellicles were washed twice with $10 \mathrm{ml}$ sterile water and then placed in $5 \mathrm{ml} 50 \mathrm{mM}$ potassium phosphate ( $\mathrm{pH} 6.0)$ containing no cellulase or $0.1 \%(\mathrm{w} / \mathrm{v})$ cellulysin cellulase (Calbiochem-Novabiochem). The pellicles were incubated on a rotating shaker (30 r.p.m.) at room temperature overnight. At least three pellicles were used for each treatment, and the experiments were repeated at least three times.

Virulence assays. One-week-old Nicotiana benthamiana seedlings were transplanted into $10 \mathrm{~cm}$ pots. Seedlings were placed in a growth chamber operating at $25{ }^{\circ} \mathrm{C}$ with $12 \mathrm{~h}$ continuous light per $24 \mathrm{~h}$ period. Light was provided by $40 \mathrm{~W}$ cool white fluorescent bulbs at $240 \mu \mathrm{mol}$ photons $\mathrm{m}^{-1} \mathrm{~s}^{-1}$. Plants were watered once daily and fertilized once per week with a $50 \%$ solution of Miracle Gro (Scotts). Plant assays were performed essentially as described by Hirano et al. (1997). Four- to six-week-old N. benthamiana leaves were infiltrated with a bacterial suspension of $10^{4}$ c.f.u. $\mathrm{ml}^{-1}$. Inoculated plants were placed in plastic bags and incubated in a growth chamber at $25^{\circ} \mathrm{C}$ throughout the course of the assay. At each sampling time, whole leaves were homogenized using a Polytron equipped with a model PTA 20 TS probe (Brinkmann Instruments) in $20 \mathrm{ml}$ sterile water, and the suspension was dilution-plated on LB medium containing appropriate antibiotics. The data were analysed using a Student's $t$ test with a $95 \%$ confidence interval. 
Table 1. Strains and plasmids used in this study

Abbreviations: $\mathrm{Ap}^{\mathrm{R}}$, ampicillin resistance; $\mathrm{Cm}^{\mathrm{R}}$, chloramphenical resistance; $\mathrm{Km}^{\mathrm{R}}$, kanamycin resistance; $\mathrm{Nx}^{\mathrm{R}}$, nalidixic acid resistance.

\begin{tabular}{|c|c|c|}
\hline Strain or plasmid & Relevant characteristics & Source or reference \\
\hline \multicolumn{3}{|l|}{ Strains } \\
\hline E. coli $\mathrm{DH} 5 \alpha$ & 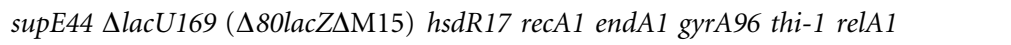 & Clontech \\
\hline \multicolumn{3}{|l|}{ Enteritidis } \\
\hline $27655-3 b$ & Clinical isolate & Collinson et al. (1991) \\
\hline$b c s A$ mutant & In-frame deletion removing nucleotides encoding amino acids $165-828$ in BcsA & White et al. (2003) \\
\hline \multicolumn{3}{|l|}{ D. dadantii } \\
\hline 3937 & Wild-type, Saintpaulia ionantha (African violet) isolate & Lemattre \& Narcy (1972) \\
\hline Nx3937 & $3937 \mathrm{Nx}^{\mathrm{R}}$ derivative & Yap et al. (2008) \\
\hline WPP472 & $\mathrm{Cm}^{\mathrm{R}}$, adrA:: cat & This work \\
\hline WPP473 & $\mathrm{Km}^{\mathrm{R}} \mathrm{Cm}^{\mathrm{R}}$, chromosomal insertion lacY-adrA-kan-prt in WPP472 & This work \\
\hline WPP101 & $\mathrm{Nx}^{\mathrm{R}} C m^{\mathrm{R}}, b c s A:: c a t$ & Yap et al. (2005) \\
\hline A1524 & $\mathrm{Cm}^{\mathrm{R}}$, pecS : : MudIIPR13 & Reverchon et al. (1994) \\
\hline \multicolumn{3}{|l|}{ Plasmids } \\
\hline pGEM-T Easy & $\mathrm{Ap}^{\mathrm{R}}$, lac $Z^{\prime}$, cloning vector & Promega \\
\hline pBluescript SK $(+/-)$ & $\mathrm{Ap}^{\mathrm{R}}$, lac $Z^{\prime}$, cloning vector & Stratagene \\
\hline pKD4 & $\mathrm{Km}^{\mathrm{R}}$, template plasmid carrying kan cassette & Datsenko \& Wanner (2000) \\
\hline pKD3 & $\mathrm{Cm}^{\mathrm{R}}$, template plasmid carrying cat cassette & Datsenko \& Wanner (2000) \\
\hline pTadrA::cat & $\mathrm{Ap}^{\mathrm{R}} \mathrm{Cm}^{\mathrm{R}}, 2.7 \mathrm{~kb}$ fragment containing adrA:: cat in pGEM-T Easy & This work \\
\hline pT $\Delta b c s C:: c a t$ & $\begin{array}{l}\mathrm{Ap}^{\mathrm{R}} \mathrm{Cm}^{\mathrm{R}}, 4.0 \mathrm{~kb} \text { fragment containing flanking regions of } b c s C \text {, cut with } \\
\text { HindIII and ligated to cat in pGEM-T Easy }\end{array}$ & This work \\
\hline pTcelY: : kan & $\mathrm{Ap}^{\mathrm{R}} \mathrm{Km}^{\mathrm{R}}, 5.1 \mathrm{~kb}$ fragment containing $c e l Y::$ kan in pGEM-T Easy & This work \\
\hline pThrpYJcat & $\begin{array}{l}\mathrm{Ap}^{\mathrm{R}} \mathrm{Cm}^{\mathrm{R}}, 5.2 \mathrm{~kb} h r p L \text {-deleted } h r p Y-h r p J \text { region on pGEM-T Easy; complete } h r p L \\
\text { coding region removed with } S m a \mathrm{I} \text { and HindIII, blunt-ended and ligated to cat }\end{array}$ & This work \\
\hline
\end{tabular}

Table 2. Oligonucleotides used in this study

\begin{tabular}{|c|c|c|c|}
\hline Primer & Sequence $\left(5^{\prime} \rightarrow 3^{\prime}\right)$ & Restriction sites* & Amplified region \\
\hline P0198 & CTGCAGGATCCGTGTAGGCTGGAGCTGCTTC & PstI, BamHI & $1 \mathrm{~kb}$ cat or $1.6 \mathrm{~kb} \mathrm{kan}$ \\
\hline P0199 & CTGCAGGATCCCATATGAATATCCTCCTTA & $\overline{P s t \mathrm{I}}$, BamHI & \\
\hline P0805 & GAACGGGTCGGGGGATCCCTGATGCATAA & BamHI & \\
\hline P0806 & GCTACCTGGAGCTGGGCGAT & - & $2.2 \mathrm{~kb}$ adrA spanning region \\
\hline P0807 & TCAGATGCTGGATCGGCGCGACGT & - & \\
\hline P0809 & CGTACTCTGCGAAGCTTCCCAGATAAAAAATAAGCGAGCCACCT & HindIII & \\
\hline P0810 & GGAAGCTTCGCAGAGTACGGCATGAGTGACCTGCAACAGCA & HindIII & $1.5 \mathrm{~kb} b c s C$ flanking region \\
\hline P0811 & TCGCCATGTGTACCGCAAGAATAAGG & - & \\
\hline P0812 & AATATCGATCAGGTTACGGTTGATTCACAGGG & - & $3.5 \mathrm{~kb} b c s D$ and $c e l Y$ spanning region \\
\hline P0813 & AAGAGGAAGTCCCCAGTACGATCAGC & - & \\
\hline
\end{tabular}

${ }^{\star}$ Restriction sites are indicated in the primer sequence by underlined or bold type. Italic type indicates the overhang used in crossover PCR. 
Immunoblot analysis. Strains were grown at $28{ }^{\circ} \mathrm{C}$ in SOBG medium for 2 days, at which point the wild-type $D$. dadantii pellicle had just completely covered the surface of the culture. Cultures were run in duplicate to obtain soluble and whole-cell lysate fractions. The pellicle was decanted for the preparation of whole-cell lysate and soluble proteins. For mutants that did not form pellicles, the SAL biofilm was harvested. Pellicles and SAL biofilms were centrifuged to harvest bacterial cells, and the pellets were resuspended in $200 \mu \mathrm{l}$ EasyLyse (Epicentre), then incubated for $10 \mathrm{~min}$ at room temperature. Soluble protein samples were briefly centrifuged and the supernatant was transferred to a fresh microcentrifuge tube. The protein concentration of both soluble and whole-cell lysate fractions was determined with a Bradford assay (Bio-Rad Laboratories). One milligram per millitre of total protein from each sample was suspended in a modified Laemmli buffer (Hammer et al., 2007), incubated at $100{ }^{\circ} \mathrm{C}$ for $10 \mathrm{~min}$, and separated in an SDS $4-20 \%$ polyacrylamide gel by electrophoresis (NuSep). Proteins were subsequently analysed by immunoblotting with HrpN-specific polyclonal antiserum (a gift from A. Collmer, Cornell University) with an ImmunoStar AP goat anti-rabbit IgG detection kit (Bio-Rad Laboratories), following the manufacturer's instructions. The immunoblot experiment was repeated three times.

Epifluorescence microscopy. Epifluorescence microscopy was performed essentially as previously reported (Yap et al., 2005). Briefly, pellicle and SAL biofilm fragments were heat-fixed to glass slides and stained with the DNA stain propidium iodide (Invitrogen) and the $\beta$-glucan stain calcofluor (Becton, Dickinson and Co.) according to the manufacturers' instructions. The stained $D$. dadantii biofilms were observed by epifluorescence microscopy with a BX60 epifluorescence microscope (Olympus America). Separate filters were used to observe cells stained with propidium iodide (excitation, $556 \mathrm{~nm}$; emission, $592 \mathrm{~nm}$ ) and $\beta$-glucans stained with calcofluor (excitation, $360 \mathrm{~nm}$; emission, $450 \mathrm{~nm}$ ). Images of the propidium iodide-stained cells and the calcofluor-stained extracellular matrix of $D$. dadantii were captured separately as monochrome images with a Magnafire camera (Optronics) and Image Pro Plus software (MediaCybernetics). The images were false-coloured and merged with Photoshop CS2 (Adobe Systems).

Scanning electron microscopy (SEM). Pellicles were removed from 3 day-old cultures by pouring the culture into $15 \mathrm{ml}$ conical tubes. The pellicles were washed twice for $3 \mathrm{~min}$ with $10 \mathrm{ml} 0.1 \mathrm{M}$ phosphate buffer ( $\mathrm{pH} 7.4$ ). Sections were fixed for $2 \mathrm{~h}$ with $1.5 \%$ glutaraldehyde and $1 \%$ tannic acid in $0.1 \mathrm{M}$ phosphate buffer. The samples were rinsed twice in $0.1 \mathrm{M}$ phosphate buffer for $3 \mathrm{~min}$ and then dehydrated by successive ethanol treatment $(30 \%$ for $10 \mathrm{~min}$, $50 \%$ for $10 \mathrm{~min}, 70 \%$ for $10 \mathrm{~min}, 95 \%$ for $10 \mathrm{~min}$, and three washes of $100 \%$ for $10 \mathrm{~min})$. Final desiccation was accomplished by criticalpoint drying (Tousimis). Specimens were mounted on aluminium stubs and sputter-coated with gold/palladium alloy. Samples were observed in a scanning electron microscope (Hitachi S5700) in the high-vacuum mode at $10 \mathrm{kV}$. The images were processed for display using Adobe Photoshop version CS2 (Adobe Systems).

\section{RESULTS}

\section{D. dadantii encodes one of the two types of cellulose synthesis gene cluster present in the Enterobacteriaceae}

Examination of sequenced Enterobacteriaceae genomes showed that two distinct cellulose synthesis gene clusters are encoded by the Enterobacteriaceae (Fig. 1). One type (group A) is homologous to the cellulose synthesis cluster encoded by G. xylinus, while the other (group B) has mainly been characterized in S. enterica and E. coli. Based on sequence similarity and operon organization, $D$. dadantii encodes a group A cellulose synthesis gene cluster, while the Enterobacteriaceae plant pathogen Pectobacterium atrosepticum and the animal pathogens S. enterica and E. coli encode group B clusters. The Enterobacteriaceae group A cellulose synthesis BcsA and BcsB proteins are encoded by two genes, whereas in G. xylinus, this is a single protein. The D. dadantii, P. atrosepticum, E. coli and S. enterica cellulose synthesis gene clusters are all located in an otherwise collinear region of the chromosome. Some Enterobacteriaceae, such as Klebsiella pneumoniae, encode both types of gene cluster in this locus, while others, such as Erwinia tasmaniensis (not shown), encode both gene clusters in a different region of the chromosome. These gene clusters are present in other Gram-negative bacteria. For example, group B gene clusters are present in Pseudomonas, Ralstonia and Burkholderia species.

The two types of cellulose synthesis gene clusters encode some homologous genes, and some genes unique to each type of cluster. Bacteria encoding the group B cluster require $b c s E F G$ for cellulose synthesis, an operon that is not present in D. dadantii or G. xylinus, and the gene $b c s D$, for example, is only present in group A clusters. All of the cellulose synthesis clusters encode an endoglucanase homologue (celY or bcsZ), but they are located in different places relative to the other cellulose synthesis genes in group A and B gene cluster operons.

\section{D. dadantii biofilms are more similar in structure to G. xylinus biofilms than to S. enterica biofilms}

We hypothesized that there were differences in the biofilm matrix among species encoding different types of cellulose synthesis gene clusters, so we examined biofilms from three different species which encode the two different types of bcs cluster. Since biofilm formation is dependent on medium components, and inducing media differ among species, we used media previously reported to induce biofilm formation for each species. We also tested G. xylinus in SOBG medium, a medium developed for $D$. dadantii biofilm studies (Yap et al., 2005). SEM images showed significant differences in pellicle structure among these three species (Fig. 2). S. enterica cells were completely enmeshed within a matrix of smooth, highly branched strands with cells located in crypts, which may have formed as a result of bacterial digestion of the surrounding matrix (Fig. 2a, b). In contrast, G. xylinus formed thick cables of cellulose, no crypts were present, and cells were sparse in the biofilm (Fig. 2c, d). The D. dadantii and G. xylinus matrices closely resembled each other, with thicker strands and fewer branches than the S. enterica matrix (Fig. 2e, f). However, the $D$. dadantii matrix fibres had the appearance of strands of beads; thus, we refer to these 
S. enterica ${ }^{K d g K} \quad{ }^{\text {hhjJ }}{ }^{d c t A}{ }^{d h h j K}$

E. coli $\underbrace{\text { ddgk }}_{28 \%} \mathrm{yhjJ}_{29 \%}^{d c t A} \underbrace{\text { yhjK }}_{26 \%}$

P. atrosepticum

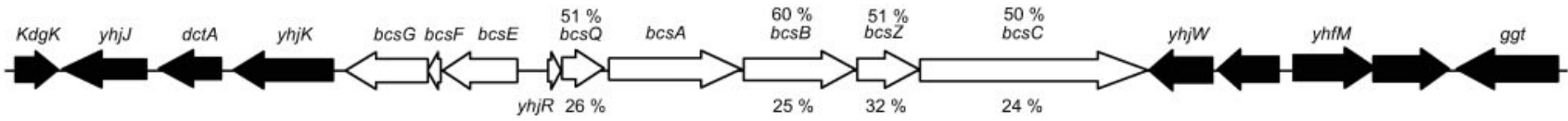

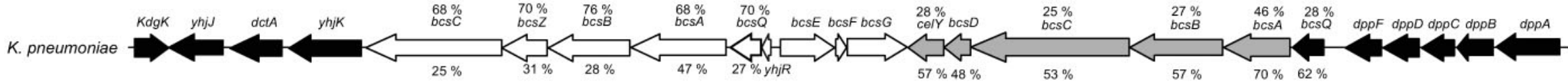

D. dadantii ${ }^{K d g K}$ ind

G. xylinus

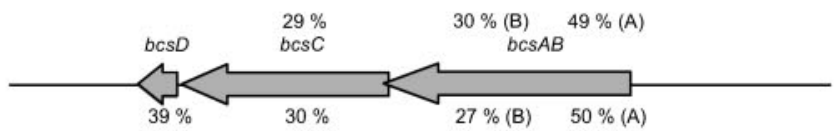

Pseudomonas syringae

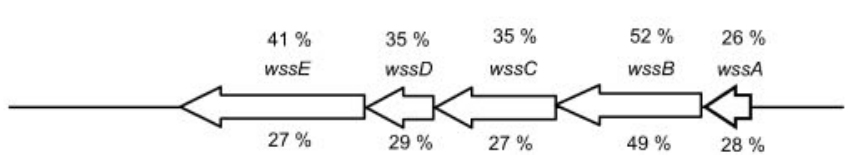

Fig. 1. Organization of the group A and group B cellulose synthesis operons. There are two classes of cellulose biosynthesis gene cluster: group A is indicated with grey arrows and group B with white arrows. Members of the Enterobacteriaceae may encode one or both of these gene clusters, typically in an otherwise collinear region of the chromosome. Numbers above the arrows indicate the amino acid identity of selected Bcs proteins compared with S. enterica LT2; numbers below the arrows indicate the amino acid identity of these proteins compared with $D$. dadantii 3937. Gene clusters encoding homologous genes are shown from the Enterobacteriaceae species $E$. coli EDL933, P. atrosepticum SCRI1043, K. pneumoniae MGH78578 and D. dadantii 3937, and from G. xylinus ATCC 23769 and Pseudomonas syringae DC3000. The P. atrosepticum SCRI 1043 bcs $A$ and E. coli EDL933 bcsQ genes have nonsense mutations near the $5^{\prime}$ ends, so amino acid identity was not included for these genes. Pseudomonas cellulose operon nomenclature differs from that of other bacteria ( $b c s Q=w s s A, b c s A=w s s B, b c s B=w s s C, b c s Z / c e / Y=w s s D$ and $b c s C=w s s E)$. 
strands as decorated cellulose nanofibres. The pellicles of both $D$. dadantii and $S$. enterica appeared to have a greater cell density than that of G. xylinus. Because the $S$. enterica cells were larger than the pore size in the biofilm lattice, the lattice would need to be digested before the cells could escape. In contrast, the $D$. dadantii cells were smaller than the holes in the cellulose lattice, and we observed $D$. dadantii cells streaming from pellicles when the pellicles were transferred from culture medium to water. S. enterica cells do not stream from pellicles when the pellicles are transferred to water.

\section{D. dadantii bcsA, bcsC and adrA mutant pellicles are resistant to cellulase}

We previously found that the cellulose synthesis mutant $b c s A$ still forms pellicles, but that these pellicles are thinner and more fragile than those formed by wild-type cells (Yap et al., 2005). Mutation of $b c s C$ showed that pellicles formed by a $b s c C$ mutant are identical to those formed by the $b c s A$ mutant (not shown). We hypothesized that the biofilm matrices formed by $b c s A$ and $b c s C$ are not composed of cellulose, and we found that the fragile pellicles produced by these strains were not degraded by cellulase, unlike pellicles formed by $D$. dadantii wild-type cultures (Fig. 3).

AdrA is required for biofilm formation by some Enterobacteriaceae. The pellicle formed by the $D$. dadanti ardA mutant appeared similar to that formed by wild-type cells macroscopically, but it was only partially degraded by cellulase (Fig. 3). Expression of adrA from a plasmid complemented this phenotype (not shown). Thus, the matrix was composed of cellulose and an additional cellulase-resistant polymer.

The pellicle formed by a celY mutant, which in all respects resembled that of the wild-type, degraded when cellulase was added, as did the G. xylinus pellicle. Notably, the $S$. enterica pellicle did not degrade when cellulase was added. Thus, although the cellulose synthase homologue $b c s A$ contributes to $S$. enterica pellicle formation, we found, as had been reported earlier, that the S. enterica pellicle is composed primarily of cellulase-resistant polymers (Solano et al., 2002).

Both FliA, a sigma factor required for flagella production, and HrpL, a sigma factor that controls the T3SS, are required for pellicle formation, but not SAL biofilm formation, in D. dadantii (Jahn et al., 2008; Yap et al., 2005). The biofilm ring formed by the $h r p L$ mutant was not degraded by cellulase (Yap et al., 2005), whereas the SAL biofilm formed by the fliA mutant was degraded (Fig. 3), suggesting that the two biofilm matrices differ.

To confirm that the lack of pellicle formation by the T3SS, fliA and $b c s$ mutants was not due to the inability of these strains to grow in pellicle-inducing medium, bacterial growth of $h r p X, h r p Y, h r p L, h r c J, b c s A, b c s C, a d r A$ and fliA mutants was measured in SOBG medium for $24 \mathrm{~h}$. The growth of the mutants in SOBG did not differ significantly from each other or from that of wild-type cells (not shown).

We stained the pellicles and SAL biofilms with calcofluor, a fluorescent dye that stains $\beta$-glucans, including cellulose, chitin and chitosan. As previously reported, the $b c s A$ mutant did not produce strands stained by calcofluor; nor did a $h r p L$ mutant, which is consistent with the resistance of these aggregates to cellulase (Yap et al., 2005). The adrA mutant produced a matrix that was stained by calcofluor, indicating that this mutant still produced cellulose, which is consistent with the partial degradation of this pellicle by cellulase (not shown).

\section{bcs $A$ and bcs $C$ are required by $D$. dadantii to produce decorated cellulose nanofibres, whereas adrA and celY are not}

We predicted that the pellicle matrix structures of the wildtype and bcs mutants would differ. SEM images of wildtype and $c e l Y$ mutant pellicles were indistinguishable (Figs $2 \mathrm{e}, \mathrm{f}$ and $4 \mathrm{a})$. In contrast, the $b c s A$ and $b c s C$ mutant pellicle matrix strands did not resemble the wild-type in decoration, branching pattern or strand width (Fig. 4c, d). Compared with the wild-type, the strands of the $b c s A$ and $b c s C$ mutants were more highly branched and irregular in width, and were not coated with uniformly spaced beads. The $D$. dadantii adrA mutant pellicle appeared to be intermediate in structure between the wild-type and the $b c s$ mutants, with beaded strands, highly branched strands, and bare strands (Fig. 4b).

\section{Although both the fliA mutant and the hrpL mutant are able to produce a SAL biofilm, the structure of the biofilm differs}

Although the T3SS sigma factor HrpL and the flagellar sigma factor FliA are required for pellicle formation across liquid surfaces, $h r p L$ and fliA mutants still form SAL biofilms (Yap et al., 2005). SEM images of the celluloseresistant biofilm ring formed by a $D$. dadantii hrpL mutant (Fig. 5a) resembled those of the $b c s A$ and $b c s C$ mutants (Fig. 4c, d). Unlike the biofilm formed by T3SS mutants, the fliA mutant biofilm is easily dislodged from the culture tube surface (Jahn et al., 2008), and it can be degraded by cellulose (Fig. 3). The matrix formed by the fliA mutant resembled that formed by wild-type cells (Fig. 5b, c, d). However, the fliA mutant cells were greatly elongated compared with the wild-type (Fig. 5d). Cells of the fliA mutant were harvested from culture media that do not induce biofilm formation, such as LB broth, and were found to resemble the wild-type (Fig. 5f, g).

D. dadantii wild-type and pellicle-deficient mutant strains sporadically produced the blue pigment indigoidine in SOBG after pellicles had formed. Indigoidine production is regulated by PecS, and mutation of pecS results in high levels of pigment production and reduced bacterial growth in culture (Reverchon et al., 1994). Indigoidine is produced 


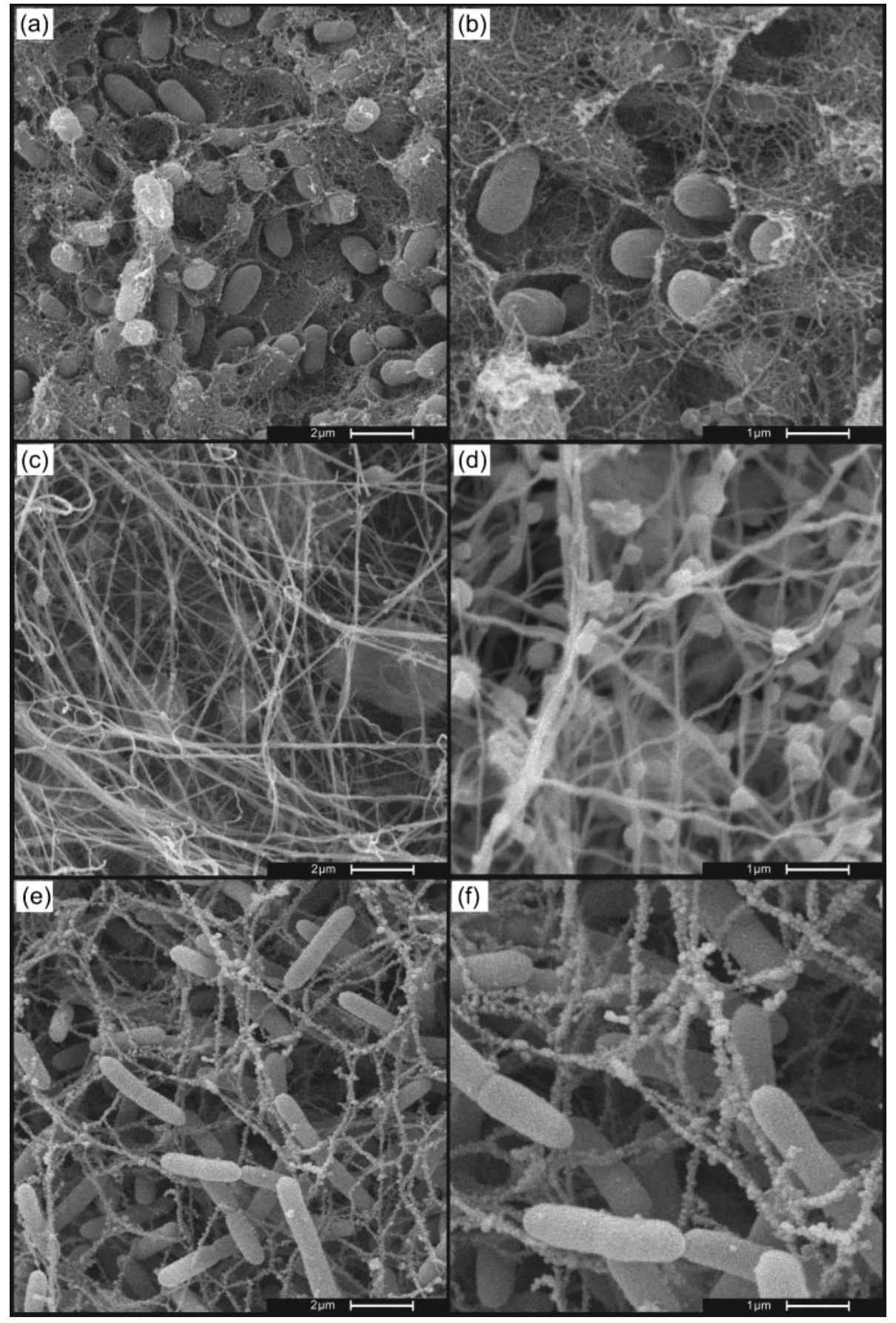

Fig. 2. Scanning electron micrographs of $S$. enterica (a, b), G. xylinus (c, d) and D. dadantii (e, f) biofilms. The images are representative of the observations from two experiments with two replicates each. $S$. enterica cells were grown in LBNS, G. xylinus cells were grown in S\&H medium (c) or SOBG (d), and D. dadantii cells were grown in SOBG. Bars: (a, c, e) $2 \mu \mathrm{m}$, (b, d, f) $1 \mu \mathrm{m}$.

by both Gram-positive and Gram-negative species, and in $D$. dadantii is thought to absorb reactive oxygen during pathogenesis (Reverchon et al., 2002; Takahashi et al., 2007). The pigment accumulated as beads that were up to twice the diameter of the bacterial cells (Fig. 5e, h).
The D. dadantii bcsA mutant is reduced in HrpN accumulation

HrpN is secreted via the T3SS, binds to the surface of planktonic $D$. dadantii cells and contributes to pellicle formation in D. dadantii (Yap et al., 2006). To determine 


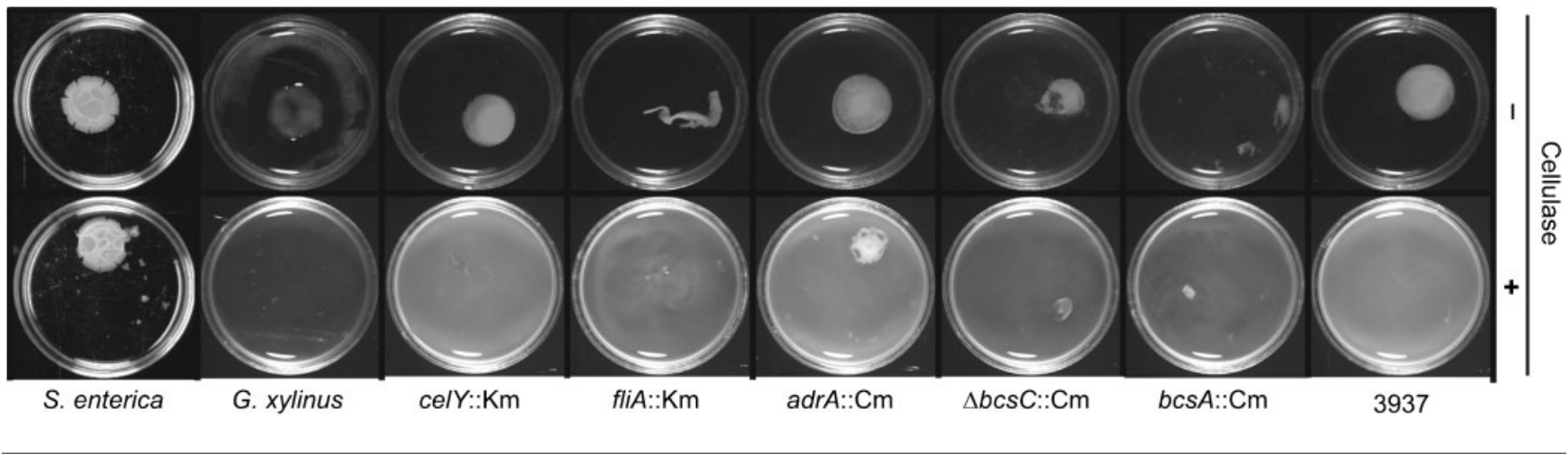

Fig. 3. Cellulase digestion of bacterial pellicles. Pellicles were removed from test tubes, placed in Petri plates containing buffer with and without cellulase, and incubated overnight. The images are representative of the observations from two to five experiments with at least two pellicles used per treatment per experiment.
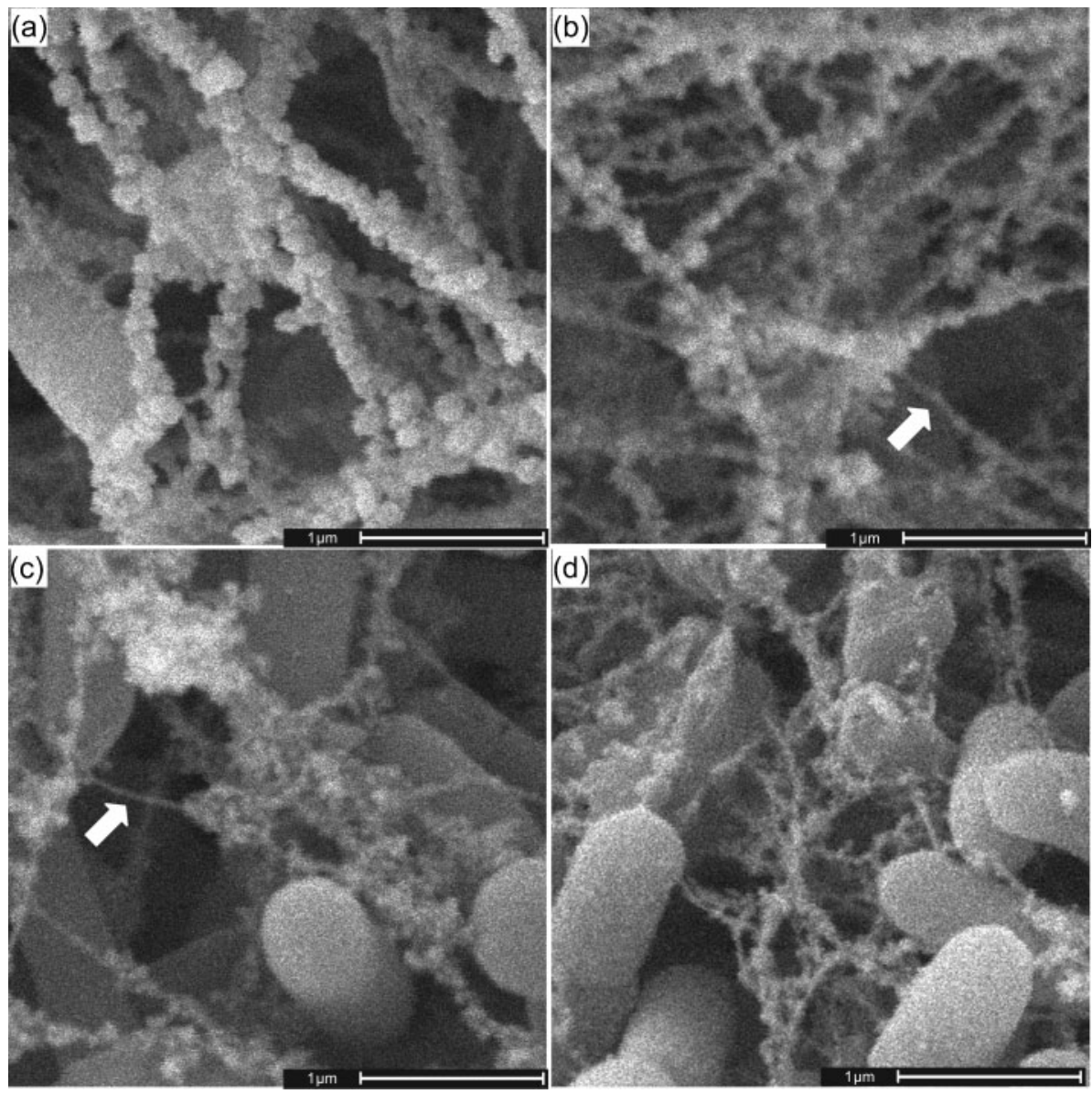

Fig. 4. Scanning electron micrographs of $D$. dadantii wild-type and cellulose gene cluster mutant strains. Biofilms of the $c e / Y$ mutant (a), adrA mutant (b), bcsA mutant (c) and bcsC mutant (d) strains. Arrows indicate examples of bare fibres produced by the $b c s A$ and $a d r A$ mutants. The images are representative of the observations from two experiments with two replicates each. Bars, $1 \mu \mathrm{m}$. 


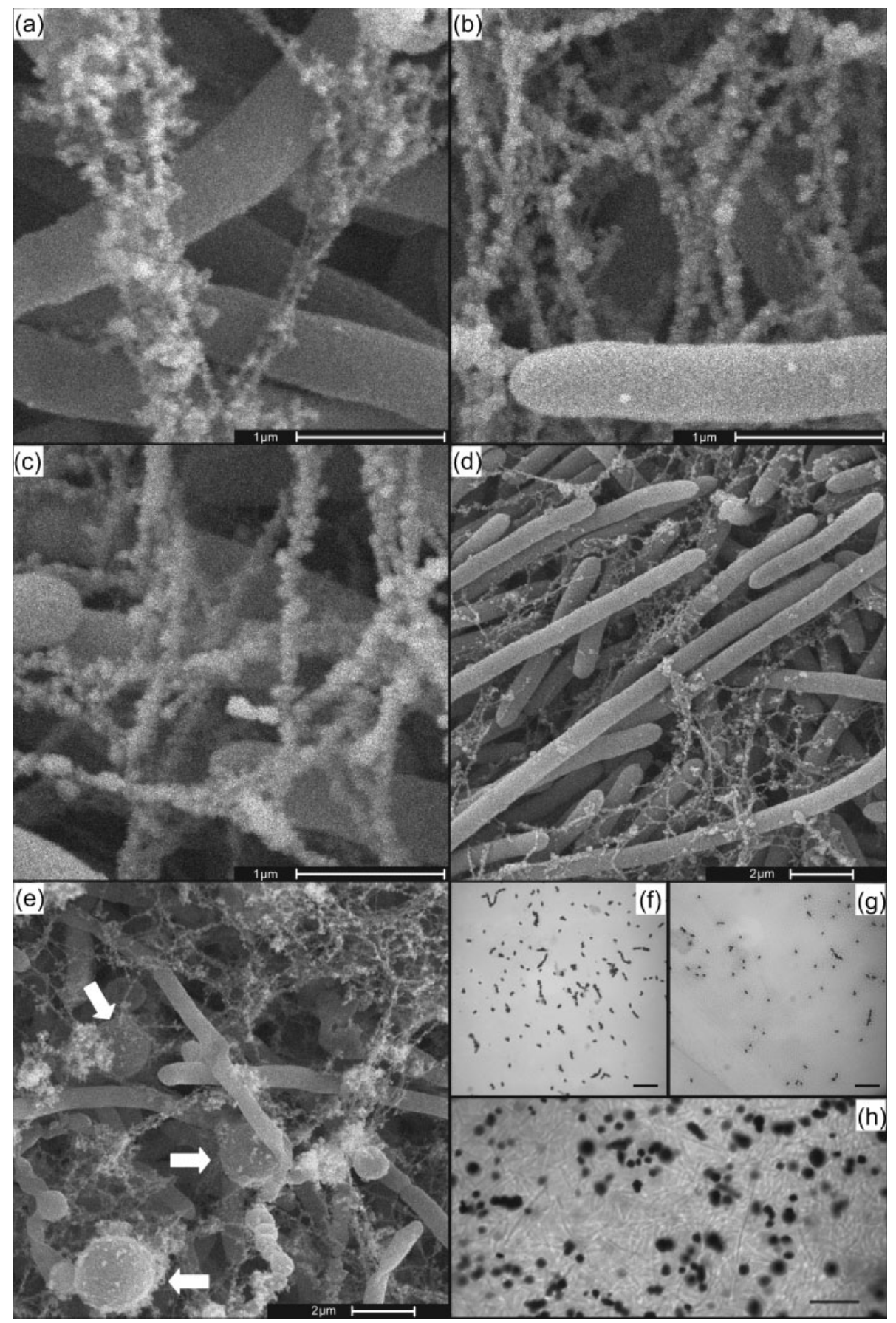

Fig. 5. Scanning electron micrographs of $D$. dadantii hrpL and fliA SAL biofilms. Biofilms of $h r p L$ mutant (a, e), fliA mutant (b, d) and wild-type (c) biofilm matrices. Bars: (a-c) $1 \mu \mathrm{m}$, (d, e) $2 \mu \mathrm{m}$. Bacterial cells of the wild-type (f) and fliA mutant (g) cells from LB broth cultures placed on slides and stained with crystal violet; bars, $10 \mu \mathrm{m}$. (h) Light micrograph of the pellicle produced by a pecS mutant; bar, $10 \mu \mathrm{m}$. In wild-type cells, PecS suppresses accumulation of indigoidine. Arrows indicate indigoidine beads. The images are representative of the observations from two experiments with two replicates each.

whether HrpN accumulation was affected in mutant strains with altered pellicle structures, total protein was harvested from pellicle and SAL biofilms, and HrpN accumulation was evaluated with immunoblots. The $b c s A$ mutant accumulated less HrpN protein than the wild-type, while the $a d r A$ mutant resembled the wild-type (Fig. 6). Thus, it is unlikely that the biofilm matrix of the bcs mutants is made of HprN protein. 


\section{D. dadantii cellulose is not required for bacterial growth in leaves}

$D$. dadantii is an effective pathogen of $N$. benthamiana and causes multiple types of symptoms in this host, including wilting, maceration and leaf curling (Jahn et al., 2008). No difference in symptom development or the growth of $b c s A$ or $a d r A$ mutant strains compared with the wild-type in $N$. benthamiana leaves was found. Thus, neither cellulose synthesis nor AdrA was required for growth of bacterial cells in N. benthamiana leaves (data not shown).

\section{DISCUSSION}

We found that $D$. dadantii cells produce decorated cellulose nanofibres unlike any previously reported cellulose fibre, and that both the T3SS and the cellulose synthesis operon are required for production of these fibres. Three gene clusters (T3SS, flagellar and cellulose synthesis) that produce extracellular proteins or polysaccharides contribute to $D$. dadantii biofilm formation (Jahn et al., 2008; Yap et al., 2005). The biofilm phenotypes of strains with mutations in these gene clusters are somewhat enigmatic. For example, although the $D$. dadantii pellicle can be completely degraded by cellulase, mutation of the cellulose synthesis operon does not eliminate pellicle formation, but rather results in the formation of a cellulase-resistant pellicle. In contrast, deletion of T3SS regulatory or structural genes eliminates both pellicle formation and cellulose synthesis (Yap et al., 2005). Deletion of $f l i A$, which controls flagellar synthesis, also eliminates pellicle formation, but not cellulose synthesis, since the SAL biofilm that forms can be degraded by cellulase.

D. dadantii encodes one of two cellulose synthesis operons found in the Enterobacteriaceae. The variation in cellulose synthesis genes is reminiscent of large gene clusters encoding other secreted macromolecules, such as $\mathrm{O}$ antigen, which are highly variable among strains or species, and which are present amidst otherwise conserved genes. Determining which, if either, group of cellulose synthesis

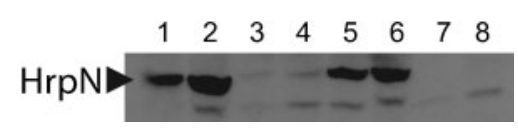

Fig. 6. Immunodectection of $\mathrm{HrpN}$ protein with rabbit polyclonal anti-HrpN. Two-day-old pellicles and SAL biofilms were centrifuged to harvest bacterial cells. Extracts were separated by $20 \%$ SDS-PAGE, and the proteins were subsequently analysed by immunoblotting with rabbit polyclonal anti-HrpN followed by conjugation of the membrane with goat anti-rabbit lgG. Lanes: 1, D. dadantii 3937 soluble protein; 2, D. dadantii 3937 whole-cell lysate; $3, b \operatorname{cs} A$ soluble protein; $4, b \operatorname{cs} A$ whole-cell lysate; 5 , adr $A$ soluble protein; 6 , adrA whole-cell lysate; 7, hrpL soluble protein; 8, hrpL whole-cell lysate. gene clusters is part of the ancestral enterobacterial genome or horizontally acquired will be complicated, since both gene clusters are present in some Enterobacteriaceae, as well as being widespread in other Gram-negative families, and it is possible that there has been recombination between these two groups of cellulose synthesis gene clusters. Regardless of the origin, the presence of solely the group A cellulose synthesis gene cluster in $D$. dadantii has resulted in a pathogen that produces a biofilm structure that differs from the enterobacterial structure typified by $S$. enterica biofilms, and which is more similar to biofilms formed by G. xylinus. The most striking structural difference between the $D$. dadantii and $S$. enterica biofilm matrices is that $S$. enterica cells are much larger than the biofilm matrix pores and are thus entrapped by the matrix, while the $D$. dadantii cells are small enough to escape their biofilm matrix. Curiously, the soft rot pathogen Pectobacterium, which is closely related to Dickeya, encodes an S. enterica-like cellulose synthesis gene cluster.

Mutation of $b c s A$ resulted in the formation of a fragile, but cellulase-resistant, pellicle. We were unable to consistently complement this mutation, so we constructed a $b c s C$ mutation in order to have another strain with a mutation in a gene homologous to a G. xylinus gene required for cellulose synthesis. The $b c s C$ mutant had the same phenotype as the bcsA mutant, supporting our hypothesis that the $D$. dadantii cellulose synthesis gene homologues are required for synthesis of a cellulose-containing pellicle, as in G. xylinus.

We hypothesize that there are regulatory ties between the D. dadantii T3SS and cellulose synthesis, and our evidence supports this hypothesis, although the mechanism remains elusive. For example, the SAL biofilm produced by the $h r p L$ mutant does not contain cellulose, and $b c s A$ mutant cells are reduced in HrpN accumulation. Unfortunately, the $D$. dadantii genome sequence provides no clues to a possible regulatory mechanism linking these two gene clusters, so it remains unknown how the cellulose synthesis operon is integrated into the $D$. dadantii regulatory network. The $b c s$ operon promoter contains no apparent binding site for HrpL, the sigma factor that activates the T3SS, nor for HrpY, a two-component system response regulator that is required for activation of HrpL (Yap et al., 2008). No obvious transcriptional regulators are encoded in the $D$. dadantii bcs operon, and AgfD, which regulates cellulose synthesis in some related enterobacteria, is not present in D. dadantii.

Recently, regulatory ties between the $D$. dadantii T3SS system and biofilm formation on plastic were found to be mediated by phosphodiesterases containing GGDEF and/or EAL motifs that may affect c-di-GMP turnover (Yi et al., 2010). The GGDEF protein AdrA is required for cellulose synthesis in some S. enterica strains (Römling et al., 2000), but we found that mutation of $a d r A$ in $D$. dadantii does not eliminate cellulose synthesis, although the $a d r A$ mutant does produce a pellicle that is morphologically different 
from that of the wild-type and more resistant to cellulase. We hypothesized that the cellulase-resistant pellicle produced by the adrA mutant might contain an altered ratio of cellulose to T3SS-secreted proteins, but the HrpN immunoassay did not support this hypothesis. However, many $D$. dadantii genes that encode motifs suggesting that they affect c-di-GMP ratios remain unexamined, and some of these may tie together regulation of the T3SS and cellulose.

The fliA and $h r p L$ mutants both produce only SAL biofilms and not pellicles, and these phenotypes are complemented by expressing fliA or $h r p L$ in the respective mutant strains (Jahn et al., 2008; Yap et al., 2005). However, the biofilms produced by these two mutants differ in composition, structure and adherence. The fliA mutant SAL biofilm is composed of cellulose, adheres poorly to the glass tube surface, and contains filamentous cells. In contrast, mutation of $h r p L$ eliminates cellulose synthesis, although the cells are similar to the wild-type in size and the biofilm adheres strongly to the glass. To date, the composition of the SAL biofilm in the hrpL mutant remains unknown, although our results suggest that elucidation of the FliA regulon may help identify the extracellular components of this SAL biofilm. We do not know the mechanism that causes the fliA mutant cells to become filamentous in the SAL biofilm, but there is a precedent for a link between flagellar regulatory genes and cell division. In the Gramnegative species Caulobacter crescentus, flagellar regulators affect the final stage of cell separation; thus, filamentous cells accumulate in the culture when regulatory genes are mutated (Muir et al., 2005).

Wild-type $D$. dadantii and all of the pellicle-deficient mutants examined sporadically produce large amounts of indigoidine when grown in pellicle-inducing medium. This insoluble blue pigment, which protects cells from oxidative damage during interactions with host plants (Reverchon et al., 2002), accumulates on the pellicle surface as beads of approximately $1-3 \mu \mathrm{m}$ in diameter. Thus, in addition to producing decorated cellulose nanofibres, $D$. dadantii can embed secondary metabolites into a cellulose matrix. Bacterial cellulose has been used as a wound dressing, and indigoidine may be useful in this application, since absorbing reactive oxygen has been reported to aid in the healing of chronic wounds (Sibbald \& Woo, 2008). Whether indigoidine is produced as insoluble beads during plant-microbe interactions or whether it becomes solubilized when bacteria grow in plants remains unknown.

The $D$. dadantii pellicle matrix is an example of an emergent property derived from two unrelated gene clusters, and it is unique among biofilm matrices and cellulose structures described to date. The decorations on the cellulose nanofibres and the accumulation of large particles of a secondary metabolite in the $D$. dadantii biofilm demonstrate novel ways that biofilm matrices can be manipulated and may lead to new industrial uses for bacterial cellulose. The role of biofilms in $D$. dadantii virulence remains unclear. This broad-host-range pathogen can attack many host species, and sometimes altered $D$. dadantii phenotypes due to gene mutations are only observed on certain plant varieties (Yang et al., 2002). Very little is known about $D$. dadantii ecology; thus, minimal information is available to develop assays for the role that biofilms may play in the survival of this pathogen in waterways or soil. Thus, much work remains to evaluate the role of biofilm formation in $D$. dadantii virulence.

\section{ACKNOWLEDGEMENTS}

This research was supported by the US Department of Agriculture (USDA) National Research Initiative (NRI) program grant 200635319-17396 and the USDA Hatch grant WIS01072 to A. O.C. This study made use of sequences from the $D$. dadantii 3937 genome project supported by USDA grant no. 2001-52100-11316 to N. T. Perna, and Pectobacterium genome sequences supported by National Science Foundation (NSF) grant no. 0412599 to A. O. C. We thank Josiah J. Hawley for technical support, and Joseph A. Heintz of the Biological and Biomaterials Preparation, Imaging, and Characterization facility at the University of Wisconsin-Madison for obtaining the SEM images.

\section{REFERENCES}

Bielecki, S., Krystynowicz, A., Turkiewicz, M. \& Kalinowska, H. (2002). Bacterial cellulose. In Biopolymers, pp. 37-90. Edited by A. Steinbuchel, E. J. Vandamne \& S. De Baets. Weinheim: Wiley-Vch.

Brown, E. E. \& Laborie, M.-P. G. (2007). Bioengineering bacterial cellulose/poly(ethylene oxide) nanocomposites. Biomacromolecules $\mathbf{8}$, 3074-3081.

Collinson, S. K., Emödy, L., Müller, K. H., Trust, T. J. \& Kay, W. W. (1991). Purification and characterization of thin, aggregative fimbriae from Salmonella enteritidis. J Bacteriol 173, 4773-4781.

Da Re, S. \& Ghigo, J. M. (2006). A CsgD-independent pathway for cellulose production and biofilm formation in Escherichia coli. J Bacteriol 188, 3073-3087.

Datsenko, K. A. \& Wanner, B. L. (2000). One-step inactivation of chromosomal genes in Escherichia coli K-12 using PCR products. Proc Natl Acad Sci U S A 97, 6640-6645.

García, B., Latasa, C., Solano, C., García-del Portillo, F., Gamazo, C. \& Lasa, I. (2004). Role of the GGDEF protein family in Salmonella cellulose biosynthesis and biofilm formation. Mol Microbiol 54, 264277.

Grenier, A. M., Duport, G., Pagès, S., Condemine, G. \& Rahbé, Y. (2006). The phytopathogen Dickeya dadantii (Erwinia chrysanthemi 3937) is a pathogen of the pea aphid. Appl Environ Microbiol 72, 1956-1965.

Hammer, N. D., Schmidt, J. C. \& Chapman, M. R. (2007). The curli nucleator protein, CsgB, contains an amyloidogenic domain that directs CsgA polymerization. Proc Natl Acad Sci U S A 104, 1249412499.

Hirano, S. S., Ostertag, E. M., Savage, S. A., Baker, L. S., Willis, D. K. \& Upper, C. D. (1997). Contribution of the regulatory gene lemA to field fitness of Pseudomonas syringae pv. syringae. Appl Environ Microbiol 63, 4304-4312.

Jahn, C. E., Willis, D. K. \& Charkowski, A. O. (2008). The flagellar sigma factor Flia is required for Dickeya dadantii virulence. Mol Plant Microbe Interact 21, 1431-1442. 
Kader, A., Simm, R., Gerstel, U., Morr, M. \& Römling, U. (2006). Hierarchical involvement of various GGDEF domain proteins in rdar morphotype development of Salmonella enterica serovar Typhimurium. Mol Microbiol 60, 602-616.

Kojima, Y., Seto, A., Tonouchi, N., Tsuchida, T. \& Yoshinaga, F. (1997). High rate production in a static culture of bacterial cellulose by a newly isolated Acetobacter strain. Biosci Biotechnol Biochem 61, $1585-1586$.

Le Quéré, B. \& Ghigo, J. M. (2009). BcsQ is an essential component of the Escherichia coli cellulose biosynthesis apparatus that localizes at the bacterial cell pole. Mol Microbiol 72, 724-740.

Lemattre, M. \& Narcy, J. P. (1972). Une infection bacterienne nouvelle du Saintpaulia due a Erwinia chrysanthemi. C R Acad Sci 58, 227-231.

Muir, R. E., Easter, J. \& Gober, J. W. (2005). The trans-acting flagellar regulatory proteins, FliX and $\mathrm{FlbD}$, play a central role in linking flagellar biogenesis and cytokinesis in Caulobacter crescentus. Microbiology 151, 3699-3711.

Phelps, M. R., Hobbs, J. R., Kilburn, D. G. \& Turner, R. F. B. (1994). Technology for regenerable biosensor probes based on enzymecellulose binding domain conjugates. Biotechnol Prog 10, 433-440.

Reverchon, S., Nasser, W. \& Robert-Baudouy, J. (1994). pecS: a locus controlling pectinase, cellulase and blue pigment production in Erwinia chrysanthemi. Mol Microbiol 11, 1127-1139.

Reverchon, S., Rouanet, C., Expert, D. \& Nasser, W. (2002). Characterization of indigoidine biosynthetic genes in Erwinia chrysanthemi and role of this blue pigment in pathogenicity. $J$ Bacteriol 184, 654-665.

Ried, J. L. \& Collmer, A. (1987). Construction and analysis of an Erwinia chrysanthemi mutant containing deletions in the pel genes encoding all of the major pectate lyase isozymes. Phytopathology 77, 1719-1719.

Römling, U., Rohde, M., Olsén, A., Normark, S. \& Reinköster, J. (2000). agfD, the checkpoint of multicellular and aggregative behaviour in Salmonella typhimurium regulates at least two independent pathways. Mol Microbiol 36, 10-23.

Sambrook, J. \& Russell, D. W. (2001). Molecular Cloning: a Laboratory Manual, 3rd edn. Cold Spring Harbor, NY: Cold Spring Harbor Laboratory.

Saxena, I. M., Lin, F. C. \& Brown, R. M., Jr (1990). Cloning and sequencing of the cellulose synthase catalytic subunit gene of Acetobacter xylinum. Plant Mol Biol 15, 673-683.

Saxena, I. M., Lin, F. C. \& Brown, R. M., Jr (1991). Identification of a new gene in an operon for cellulose biosynthesis in Acetobacter xylinum. Plant Mol Biol 16, 947-954.

Saxena, I. M., Kudlicka, K., Okuda, K. \& Brown, R. M., Jr (1994). Characterization of genes in the cellulose-synthesizing operon (acs operon) of Acetobacter xylinum: implications for cellulose crystallization. J Bacteriol 176, 5735-5752.
Sharp, D., Forsythe, S. \& Davis, J. J. (2008). Carbon fibre composites: integrated electrochemical sensors for wound management. J Biochem 144, 87-93.

Sibbald, R. G. \& Woo, K. Y. (2008). The biology of chronic foot ulcers in persons with diabetes. Diabetes Metab Res Rev 24 (Suppl. 1, ), S25-S30.

Solano, C., Garcia, B., Valle, J., Berasain, C., Ghigo, J. M., Gamazo, C. \& Lasa, I. (2002). Genetic analysis of Salmonella enteritidis biofilm formation: critical role of cellulose. Mol Microbiol 43, 793-808.

Standal, R., Iversen, T. G., Coucheron, D. H., Fjaervik, E., Blatny, J. M. \& Valla, S. (1994). A new gene required for cellulose production and a gene encoding cellulolytic activity in Acetobacter xylinum are colocalized with the bcs operon. J Bacteriol 176, 665-672.

Svensson, A., Nicklasson, E., Harrah, T., Panilaitis, B., Kaplan, D. L., Brittberg, M. \& Gatenholm, P. (2005). Bacterial cellulose as a potential scaffold for tissue engineering of cartilage. Biomaterials 26, 419-431.

Tabuchi, M. (2007). Nanobiotech versus synthetic nanotech? Nat Biotechnol 25, 389-390.

Takahashi, H., Kumagai, T., Kitani, K., Mori, M., Matoba, Y. \& Sugiyama, M. (2007). Cloning and characterization of a Streptomyces single module type non-ribosomal peptide synthetase catalyzing a blue pigment synthesis. J Biol Chem 282, 9073-9081.

Tsioptsias, C. \& Panayiotou, C. (2008). Preparation of cellulosenanohydroxyapatite composite scaffolds from ionic liquid solutions. Carbohydr Polym 74, 99-105.

White, A. P., Gibson, D. L., Collinson, S. K., Banser, P. A. \& Kay, W. W. (2003). Extracellular polysaccharides associated with thin aggregative fimbriae of Salmonella enterica serovar Enteritidis. J Bacteriol 185, 5398-5407.

Wiegand, C., Elsner, P., Hipler, U.-C. \& Klemm, D. (2006). Protease and ROS activities influenced by a composite of bacterial cellulose and collagen type I in vitro. Cellulose 13, 689-696.

Yang, C.-H., Gavilanes-Ruiz, M., Okinaka, Y., Vedel, R., Berthuy, I., Boccara, M., Chen, J. W. T., Perna, N. T. \& Keen, N. T. (2002). hrp genes of Erwinia chrysanthemi 3937 are important virulence factors. Mol Plant Microbe Interact 15, 472-480.

Yap, M.-N., Yang, C. H., Barak, J. D., Jahn, C. E. \& Charkowski, A. O. (2005). The Erwinia chrysanthemi type III secretion system is required for multicellular behavior. J Bacteriol 187, 639-648.

Yap, M.-N., Rojas, C. M., Yang, C.-H. \& Charkowski, A. O. (2006). Harpin mediates cell aggregation in Erwinia chrysanthemi 3937. J Bacteriol 188, 2280-2284.

Yap, M. N., Yang, C.-H. \& Charkowski, A. O. (2008). The response regulator HrpY of Dickeya dadantii 3937 regulates virulence genes not linked to the hrp cluster. Mol Plant Microbe Interact 21, 304-314.

Yi, X., Yamazaki, A., Biddle, E., Zeng, Q. \& Yang, C.-H. (2010). Genetic analysis of two phosphodiesterases reveals cyclic diguanylate regulation of virulence factors in Dickeya dadantii. Mol Microbiol 77, 787-800.

Edited by: I. K. Toth 